\title{
Expression of cyclin B1 after induction of senescence and cell death in non-small cell lung carcinoma A549 cells
}

\author{
Agnieszka Żuryń1, Maciej Gagat ${ }^{1}$, Aleksandra Antonina Grzanka², \\ Lidia Gackowska ${ }^{3}$, Alina Grzanka ${ }^{1}$ \\ ${ }^{1}$ Department of Histology and Embryology, Nicolaus Copernicus University in Torun, \\ Collegium Medicum in Bydgoszcz, Poland \\ ${ }^{2}$ Department of Dermatology and Sexually transmitted Diseases, Nicolaus Copernicus University \\ in Torun, Collegium Medicum in Bydgoszcz, Poland \\ ${ }^{3}$ Department of Immunology, Nicolaus Copernicus University in Torun, Collegium Medicum \\ in Bydgoszcz, Poland
}

\begin{abstract}
The purpose of this study was to evaluate the level of mitotic cyclin B1 in the context of senescence and cell death in A549 non-small cell lung carcinoma cells. This was performed through analysis of the cell cycle, the percentage of SA- $\beta$-galactosidase-positive, as well as TUNEL-positive cells. Morphological alterations were studied using a transmission electron microscope. Changes in the intracellular level and the presence of cyclin B1 in the nucleus and cytoplasm areas were detected by flow cytometry and confocal fluorescence microscopy, respectively. In the cells exposed to various concentrations of doxorubicin, different kinds of cell death and senescent phenotype were observed. Alterations in the cell cycle and increased polyploidy may be indicative of mitotic catastrophe execution. Changes in cyclin B1 may also be strictly related to its different regulation at mitotic catastrophe and senescence programs. (Folia Histochemica et Cytobiologica 2012, Vol. 50, No. 1, 58-67)
\end{abstract}

Key words: cyclin B1, A549, senescence, cell death

\section{Introduction}

Cyclin-dependent kinases (CDKs) mediate cell cycle progression by phosphorylating different substrates at specific cell cycle phases. Through proper synthesis and regulation of cyclins, CDKs are activated in a controlled manner.

Correspondence address: A. Grzanka,

Department of Histology and Embryology, Nicolaus

Copernicus University, Collegium Medicum in Bydgoszcz,

Karlowicza Str. 24, 85-092 Bydgoszcz, Poland;

tel.: + 48525853725 , fax: + 48525853734 ;

e-mail: agrzanka@cm.umk.pl
Cyclin B1 forms with cdc2 (Cdk1) a complex called 'mitotic promoting factor' (MPF) that is crucial for G2/M transition [1]. Thus, insufficient levels of cyclin B-Cdk1 complexes, e.g. as a result of attenuation of cyclin B1 promoter by p53, are related to cell cycle arrest at G2 [2]. Production of cyclin B1 in the cytoplasm during S-phase is followed by its transport to the nucleus at late G2 phase and, finally, its subsequent removal during anaphase via an ubiquitin-related pathway [3].

There are reports suggesting that changes in the regulation of cyclin expression may be involved not only in unrestrained cell growth and malignant transformation, but also in tumor suppressor mechanisms [4]. Exposure to anti-cancer drugs can cause not only apoptosis but also non-apoptotic mechanisms inducing cell death such 
as senescence and mitotic catastrophe. A hypermitogenic cell cycle arrest in G1 or G2 may be observed after induction of senescence in cancer cells [5]. Stress-induced premature senescence is independent of telomere length and could result from the action of anticancer drugs. There are several symptoms of senescence on the cellular level, including flattened and enlarged cell shape, increased cytoplasmic granularity, lipofuscin accumulation, enhanced senescence-associated $\beta$-galactosidase activity, as well as changes in expression of the critical cell cycle regulatory tumor suppressor genes [610]. From these genes, p53 and p16 are the most commonly mutated in different types of cancer and they are implicated in the execution of senescence program via different transduction pathway.

Another common tumor suppressor mechanism activated by anticancer drugs is apoptotic cell death. It consists of biochemical and morphological alterations leading to removal of cells without induction of inflammation. Mitotic catastrophe is generally considered to be a kind of cell death resulting from mitotic failure that can be accompanied by micronucleation and multinucleation; but on the other hand, some polyploid cells may survive and undergo depolyploidization. Cell death as a result of abnormal mitosis may be related to premature activity of cyclin B1/Cdk1 in the nucleus area and chromatin condensation. Moreover, there are some suggestions that cyclin B1 is crucial for the execution of mitotic catastrophe [11]. However, little is yet known about changes in the expression of cyclin $\mathrm{B} 1$ in the process of senescence.

In this work, we would like to verify if changes in cyclin B1 level might be related to the regulation of senescence and/or cell death programs induced by doxorubicin in A549 cells.

\section{Material and methods}

Cell culture and treatment. The human non-small cell lung carcinoma cell line A549 was kindly provided by P. Kopinski, Ph.D. (Department of Gene Therapy, Ludwik Rydygier Collegium Medicum in Bydgoszcz, Nicolaus Copernicus University in Torun, Poland). The cells were cultured in monolayers at $37^{\circ} \mathrm{C}$ in a humidified $\mathrm{CO}_{2}$ incubator $(5 \%$ $\mathrm{CO}_{2}$ ) in DMEM (Gibco) with the addition of $10 \%$ fetal bovine serum (FBS; Gibco) and $50 \mu \mathrm{g} / \mathrm{ml}$ of gentamycin (Sigma-Aldrich). Doxorubicin (Adriblastin PFS; Pharmacia Italia S.p.A., Pfizer Group) was diluted in sterile water and was added to the cell culture in appropriate doses. Twenty-four hours after seeding, the cells were exposed to doxorubicin $(50,100,200 \mathrm{nM})$ for $72 \mathrm{~h}$. Thereafter, the cells were incubated in a drug-free medium for a further $72 \mathrm{~h}$, and the subsequent experimental procedures were performed.
Cell cycle analysis. The cell pellet obtained after trypsinization and washing with PBS was suspended in $1 \mathrm{ml}$ of NSS solution $(50 \mu \mathrm{g} / \mathrm{ml}$ PI, $0.0112 \%$ (w/v) sodium citrate (Sigma-Aldrich); $0.03 \%$ (v/v) nonylphenylpolyethylene glycol (Nonidet P40 Substitute; Fluka). The cells were centrifuged $(5 \mathrm{~min}$ at $300 \times \mathrm{g}$ ) and resuspended in $250 \mu \mathrm{l}$ of NSS. After incubation in the dark, (15 min, RT), the cells were incubated in the same conditions in the presence of RNase A solution $(250 \mu \mathrm{l} ; 10 \mu \mathrm{g} / \mathrm{ml}$ RNase A in PBS) (Sigma-Aldrich). Thereafter, $0.5 \mathrm{ml}$ PBS was added and the cells were analyzed using a flow cytometer (FACScan; Becton-Dickinson). Cell cycle distribution was estimated using CellQuest software (Becton-Dickinson). For each sample, 20,000 events were acquired and the nonclumped cells were gated for further measurements.

TUNEL assay. DNA fragmentation was measured by the terminal deoxynucleotidyl transferase-mediated dUTP nickend labeling (TUNEL) method using a commercially available kit (APO-DIRECT, BD Biosciences Pharmingen), according to the manufacturer's instructions as described by Litwiniec [6].

Senescence-associated $\boldsymbol{\beta}$-galactosidase assay. Senescence-associated $\beta$-galactosidase activity was determined using the Senescent Cells Staining Kit (Sigma-Aldrich), according to the manufacturer's protocols. Briefly, after fixation procedure, the cells were incubated overnight in the presence of a staining solution containing X-gal (5-bromo-4-chloro-3-indolyl-b-galactopyranoside) in the dark $\left(37^{\circ} \mathrm{C}\right)$. The percentage of blue-stained cells was determined by light microscopy (Eclipse E800, Nikon). Results were presented as the mean number of SA- $\beta$-galactosidase positive cells per 100 cells.

Transmission Electron Microscopy (TEM). Conventional electron microscopy was used to visualize A549 morphology at the ultrastructural level. The control and treated cells (day 3 ) were harvested by trypsinization, washed with PBS and fixed in 3.6\% glutaraldehyde (pH 7.2, Merck) (30 min, RT). After washing in $0.1 \mathrm{M}$ sodium cacodylate buffer ( $\mathrm{pH} 7.2$, Sigma-Aldrich), the cells were postfixed in $2 \%$ buffered OsO4 for $2 \mathrm{~h}$, dehydrated in ethanol (40-100\%), and embedded in Epon E812. Semithin sections were stained with $1 \%$ toluidine blue and used for targeting the cells. Ultrathin sections (40 nm thick) were double-stained with uranyl acetate (Chemapol, Prague, Czech Republic) and lead citrate (BDH, Poole, UK). The material was examined using a transmission electron microscope JEM 100 CX (JEOL, Tokyo, Japan).

Flow cytometric analysis of cyclin B1 expression. Cells grown on six-well plates were trypsinized, washed with PBS, centrifuged $(5 \mathrm{~min}, 300 \times \mathrm{g})$ and resuspended at a final concentration of $1-2 \times 10^{6} \mathrm{cells} / \mathrm{ml}$ in $1 \mathrm{ml}$ PBS with the addition of $100 \mu \mathrm{l}$ of formaldehyde (Polysciences, Inc). After incubation on ice ( $15 \mathrm{~min}$ in the dark) and subsequent 
A

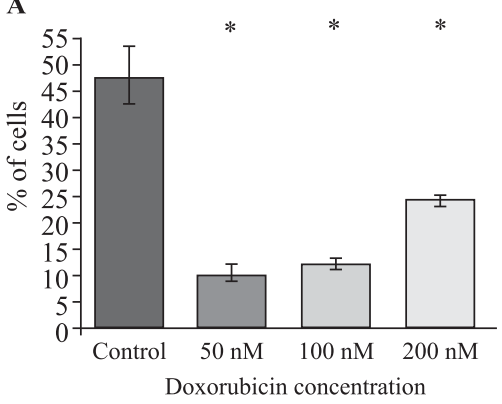

D

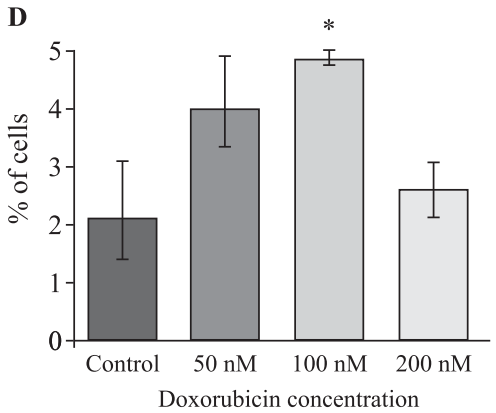

B

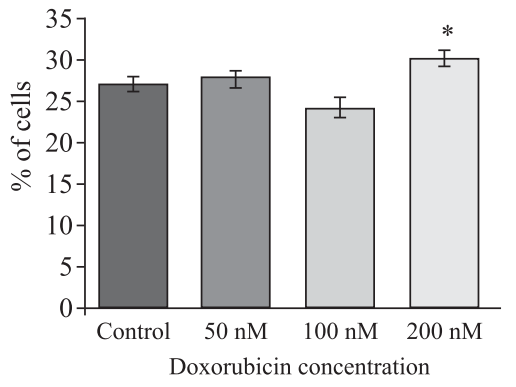

C

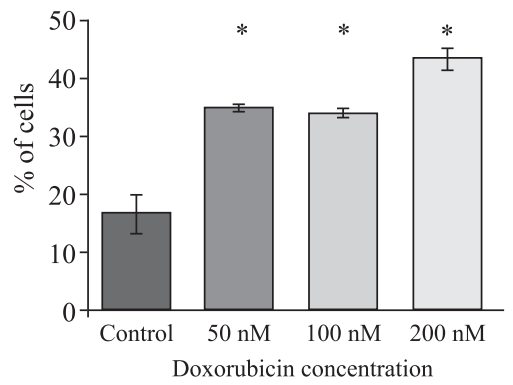

E

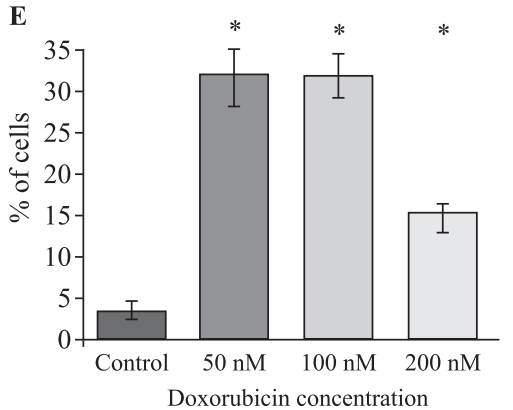

Figure 1. Flow cytometry analysis of the cell cycle distribution of A549 cells stained with RNase/PI. A diagram for each of the groups defined according to DNA content is shown. A: Cells with DNA content corresponding to G0/G1 phases. B: Cells with DNA content corresponding to S phase. C: Cells with DNA content corresponding to G2/M phases. D: Cells with DNA content corresponding to subG1 fraction. E: Cells with DNA content corresponding to polyploidy. Columns - median percentage of cells; bars — interquartile range. Asterisks denote statistical significance compared to control cells $(\mathrm{p}<0.05)$. Results are representative of five independent experiments

centrifugation $(5 \mathrm{~min}, 300 \times \mathrm{g})$, the cell pellet was permeabilized by the addition of $2 \mathrm{ml}$ of ice-cold $50 \%(\mathrm{v} / \mathrm{v})$ methanol (JT Baker). Then cells were incubated for $15 \mathrm{~min}$ on ice, washed twice with cold PBS, and resuspended in $100 \mu \mathrm{l}$ of PBS. For intracellular staining, the cell suspensions were transferred into flow cytometric tubes containing $20 \mu \mathrm{l}$ of mouse monoclonal cyclin B1 antibody: sc-7393 (Santa Cruz Biotechnology, Inc) and $20 \mu$ l of normal mouse IgG1 Alexa Fluor 488: sc-3890 (Santa Cruz Biotechnology, Inc). Following a 30-min incubation on ice and washing with PBS, the cells were centrifuged $(5 \mathrm{~min}, 500 \times \mathrm{g})$ to wash off excess antibody, and were resuspended in $200 \mu \mathrm{l}$ of PBS for flow cytometric analysis on FACScan (Becton-Dickinson), according to the manufacturer's instructions, with some modifications as described by Litwiniec [6].

CellQuest software (Becton-Dickinson) was used to calculate the percentage of cyclin B1-positive cells and the mean fluorescence intensity of these cells for each experimental case.

Immunofluorescence assay. Cells on coverslips were briefly washed with PBS, fixed in 4\% paraformaldehyde (15 min, RT) and then washed with PBS $(3 \times 5 \mathrm{~min})$. After that, the cells were incubated in permeabilization solution $(0,1 \%$ Triton $\mathrm{X}-100$ in PBS) and blocked with 1\% BSA. After permeabilization, the cells were incubated with mouse monoclonal cyclin B1 antibody: sc-245 (Santa Cruz Biotechnology, Inc) (45 min, RT), washed three times with PBS and incubated with anti- mouse IgG TRITC conjugated antibody (Sigma-Aldrich) (45 min, RT, in the dark). Nuclear staining was performed with DAPI (Sigma-Aldrich). After incubation, the cells were washed with PBS and then mounted on slides in Aqua Poly/ /Mount (Polysciences, Inc). Both cyclin B1 and DAPI staining were examined using confocal microscopy (C1, Nikon).

Statistical analysis. The analysis was performed using statistical software (GraphPad Prism, San Diego, CA, USA). The data was compared by the nonparametric Mann-Whitney $U$ test, and the changes were considered statistically significant at the level of $\mathrm{p}<0.05$.

\section{Results}

\section{Cell cycle}

As cell cycle distribution may reflect cellular response to drug treatment, we determined the percentage of cells in each phase of the cycle. The percentage of cells in the G0/G1 phases decreased in comparison with the control cells, although the tendency of the percentage seemed to increase dose-dependently (Figures 1A, 2). On the other hand, the fraction of cells with DNA content typical of S phase of the cell cycle did not change statistically after treatment, apart from the highest concentration of the doxorubicin 


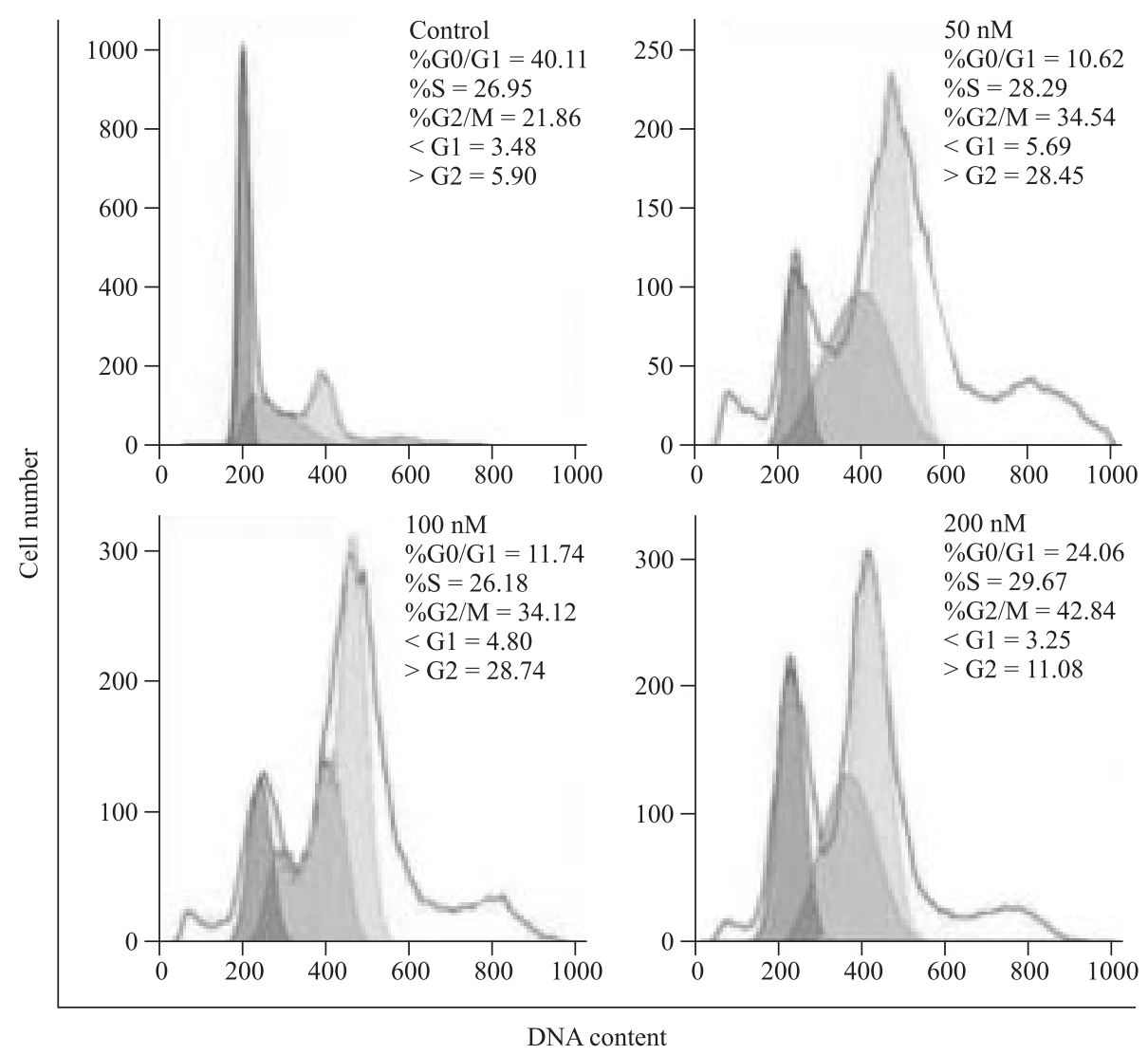

Figure 2. Flow cytometry analysis of the cell cycle distribution of A549 cells stained with RNase/PI. Representative histograms are shown: upper left — control; upper right $-50 \mathrm{nM}$ of doxorubicin; lower left $-100 \mathrm{nM}$ of doxorubicin; and lower right $-200 \mathrm{nM}$ of doxorubicin. According to DNA content, five categories of cells were distinguished: 1) cells with DNA content corresponding to G0/G1 phase; 2) cells with DNA content corresponding to S phase; 3) cells with DNA content corresponding to G2/M phase; 4) cells with fractional subG1 phase; and 5) cells with polyploid DNA content

$(200 \mathrm{nM})$, at which the percentage increased (Figures 1B, 2) (Mann-Whitney U, p < 0.05).

As regards G2/M phases, we observed an increase in the percentage of cells with DNA content typical of these phases; this increase was most visible at the highest dose of doxorubicin (Figures $1 C, 2)$. The subG1 fraction, which represents events indicative of DNA fragmentation, seemed to increase as compared with the population of control cells, but the difference was statistically significant only at $100 \mathrm{nM}$ concentration of doxorubicin (Figures 1D, 2). Similarly, the percentage of polyploidy was visibly higher after drug treatment; however, the tendency decreased at the highest concentration of doxorubicin in comparison with lower concentrations of doxorubicin used in the study (Figures $1 \mathrm{E}, 2)$.

\section{Senescence and cell death}

In order to study the effect of doxorubicin on the A549 cells, we analyzed cell survival using the trypan blue ex-

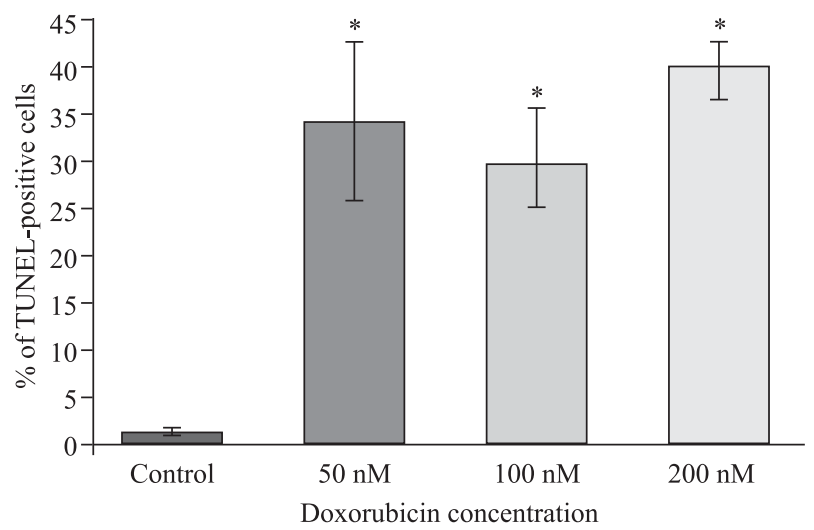

Figure 3. Flow cytometry analysis of DNA fragmentation using the TUNEL method. Columns - median percentage of TUNEL-positive cells; bars - interquartile range. Asterisks denote statistical significance compared to control cells $(p<0.05)$. Results are representative of five independent experiments

clusion dye method, the activity of SA- $\beta$-galactosidase, DNA fragmentation and cell ultrastructure in TEM. 


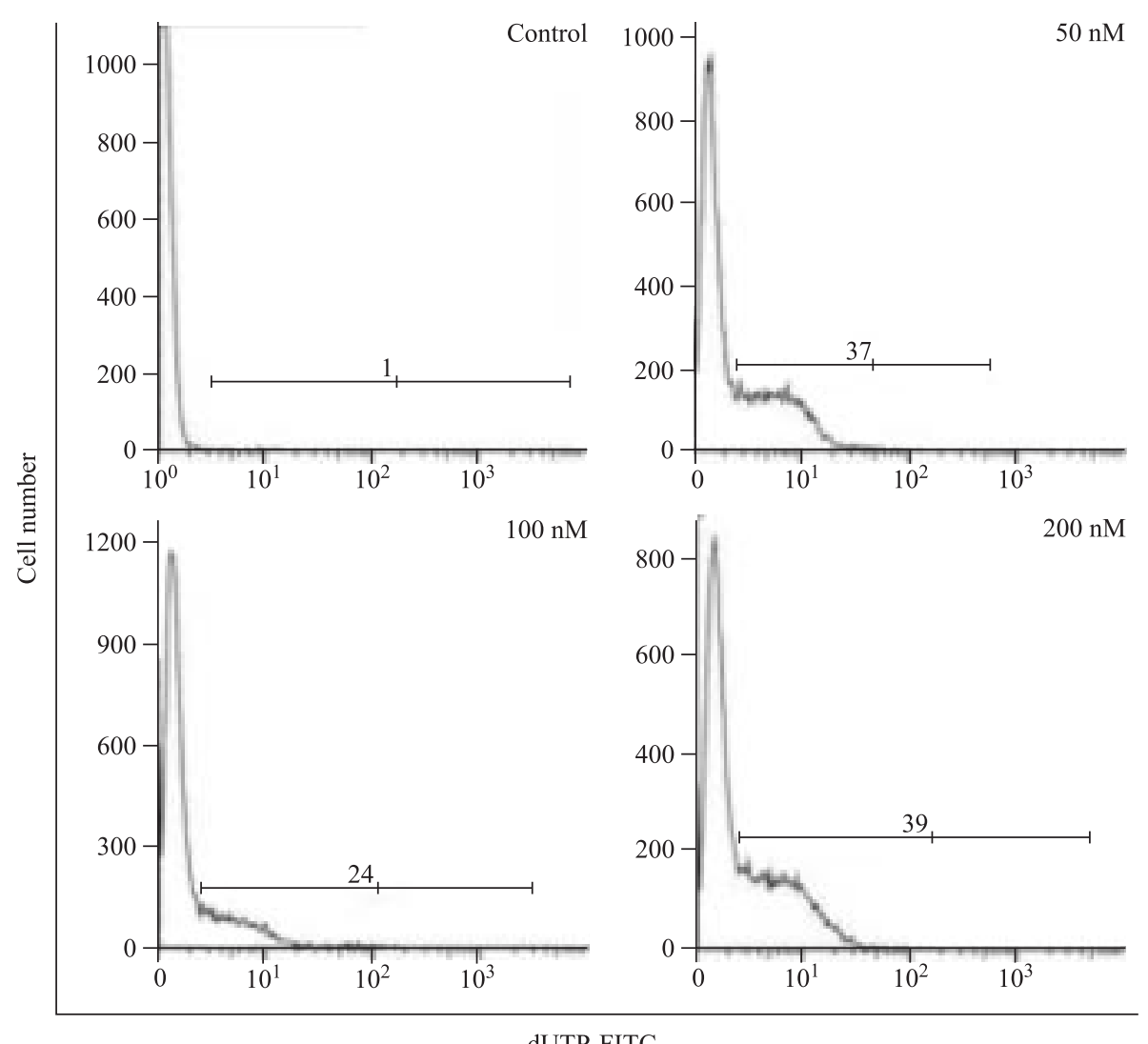

Figure 4. Flow cytometry analysis of DNA fragmentation using the TUNEL method. Representative histograms are shown: upper left - control; upper right $-50 \mathrm{nM}$ of doxorubicin; lower left $-100 \mathrm{nM}$ of doxorubicin; and lower right $-200 \mathrm{nM}$ of doxorubicin. The percentage of TUNEL-positive cells is shown on horizontal lengths

The analysis of DNA fragmentation by TUNEL method revealed that the fraction of cells with DNA single or double strand breaks was significantly higher after the treatment compared to the control cells (Figure 3), although a tendency of this increase was not dose-dependently elevated. The highest fraction of TUNEL-positive cells was obtained for $200 \mathrm{nM}$ doxorubicin (Figure 4). On the other hand, results of the trypan blue exclusion dye method showed that the viability of the cells decreased gradually (dose-dependently) after doxorubicin treatment compared to the control cells (Figure 5). Similarly, we observed enhanced SA- $\beta$-galactosidase activity reflected by the increased percentage of SA- $\beta$-galactosidase-positive cells at 50,100 and $200 \mathrm{nM}$ doxorubicin (Figure 6).

There were also observed changes in morphology of the cell revealed at the ultrastructural level. These changes were related to alterations in nuclear and cytoplasmic compartments, such as: increased volume of the cell, large amount of electron-dense and electrontransparent structures including lamellar bodies, lipofuscin-like and vacuole-like vesicles as well as disorganization of some cellular membrane systems (ER, AG)

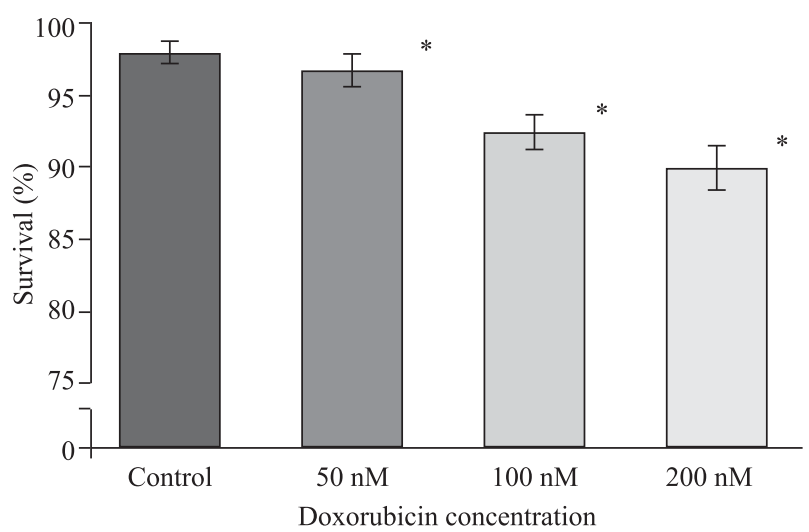

Figure 5. The effects of doxorubicin doses on cell viability. Columns - median percentage of survival cells; bars - interquartile range. Asterisks denote statistical significance compared to control cells $(\mathrm{p}<0.05)$. Results are representative of ten independent experiments

and appearance of abnormally-shaped nuclei which may be indicative of mitotic catastrophe (Figure 7). Apart from that, we observed few intranuclear inclusions. However, typical senescence-associated heterochromatin foci were not visible in our experiment. 

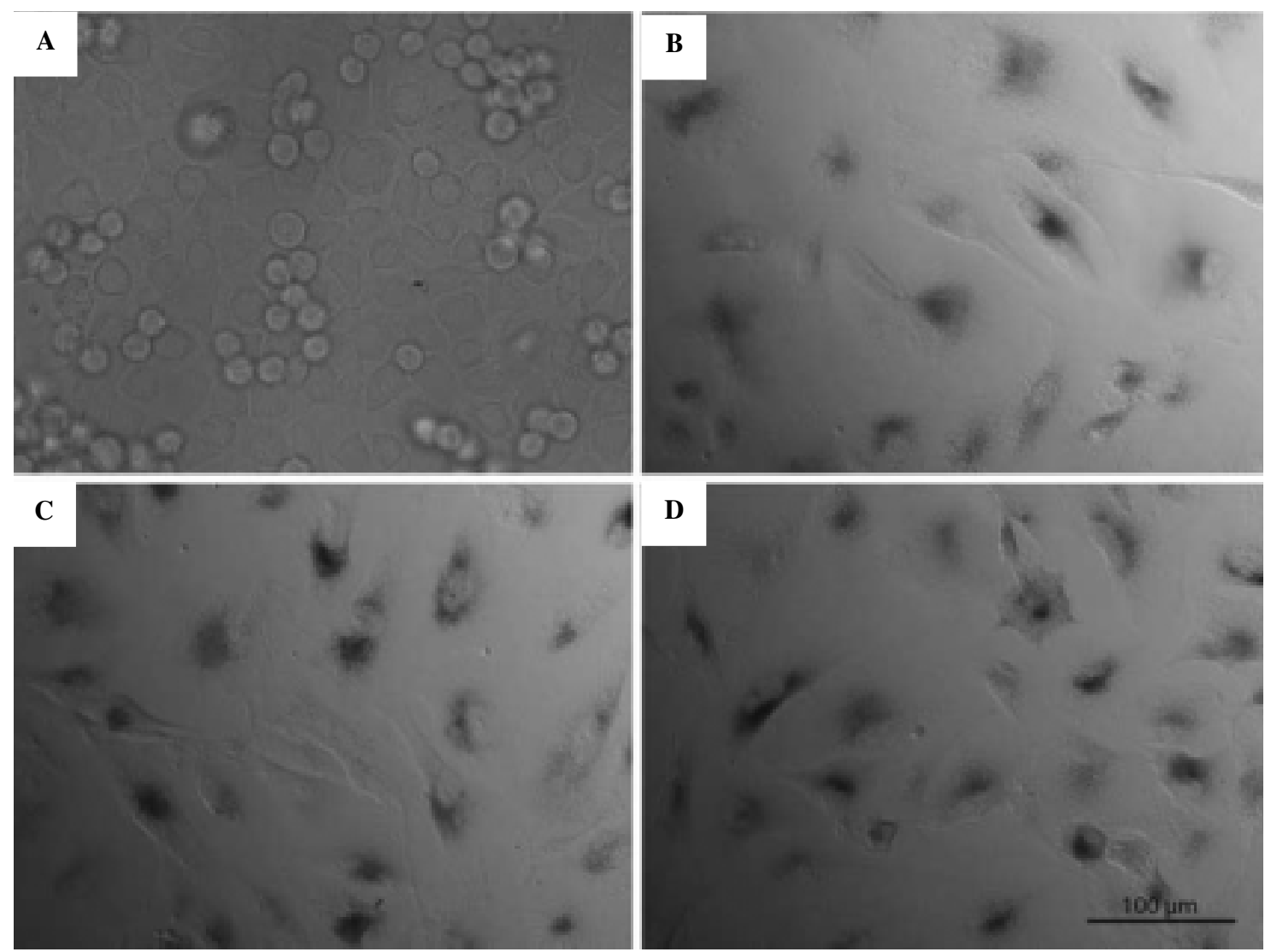

Figure 6. Activity of SA- $\beta$-galactosidase in A549 cells treated with doxorubicin. A: Control cells. B: Cells treated with $50 \mathrm{nM}$ doxorubicin. C: Cells treated with $100 \mathrm{nM}$ doxorubicin. D: Cells treated with $200 \mathrm{nM}$ doxorubicin. Changes in morphology are shown. Visible increase in volume of the cells $(C, D)$ in comparison with the control cells (A).

An enhanced activity of SA- $\beta$-galactosidase in the cells exposed to doxorubicin $(\mathrm{B}, \mathrm{C}, \mathrm{D})$ in comparison with the control cells (A). Results are representative of ten independent experiments
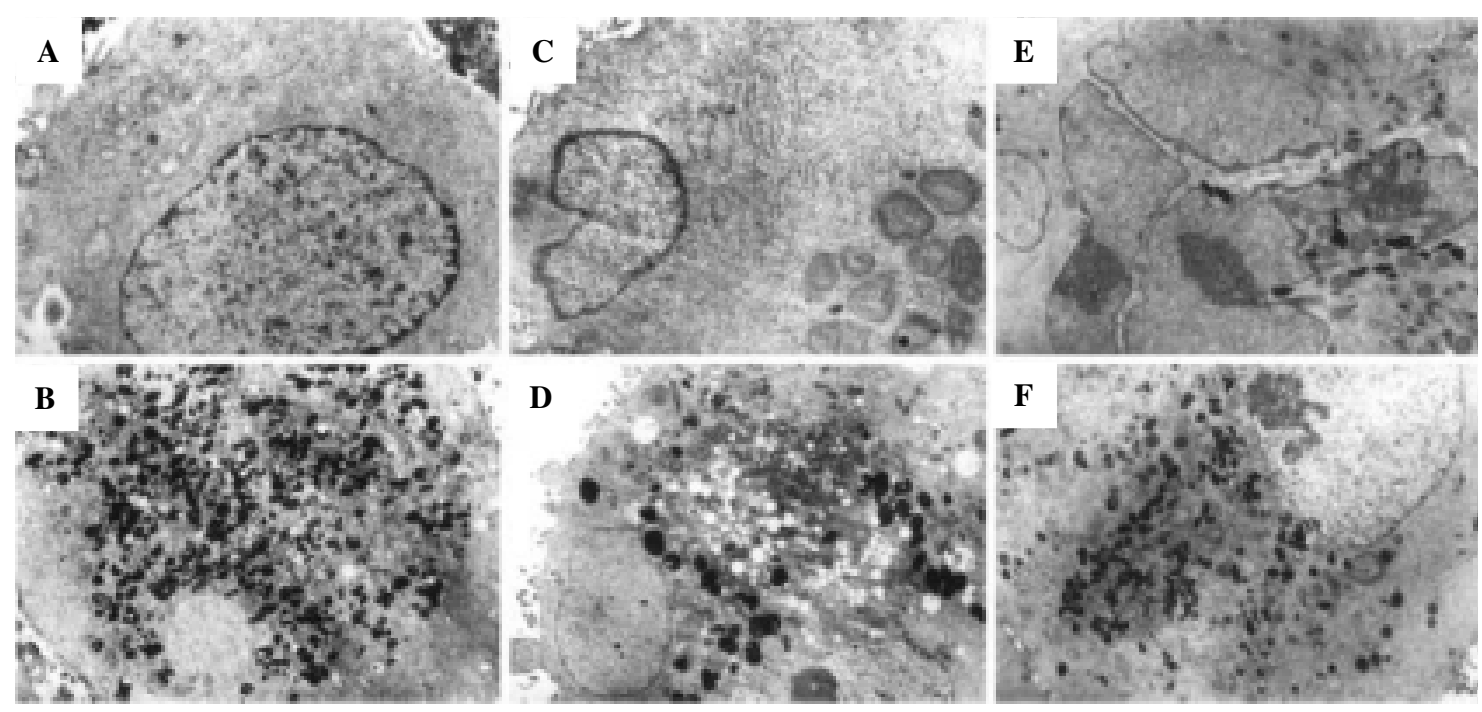

Figure 7. The ultrastructure of A549 cells treated with doxorubicin. A: Control cells. B: Cells treated with $50 \mathrm{nM}$ doxorubicin. C, D: Cells treated with $100 \mathrm{nM}$ doxorubicin. E, F: Cells treated with $200 \mathrm{nM}$ doxorubicin. Alterations of the cytoplasm and cell nucleus are shown: large amount of electron-dense (B, D, F) and electron-transparent (D) structures including lamellar bodies (C), lipofuscin-like and vacuole-like vesicles (D) as well as disorganization of some cellular membrane systems (ER, AG) (C) and appearance of abnormally-shaped nuclei (C, E). Results are representative of five independent experiments. Bar $=1 \mu \mathrm{m}$ 


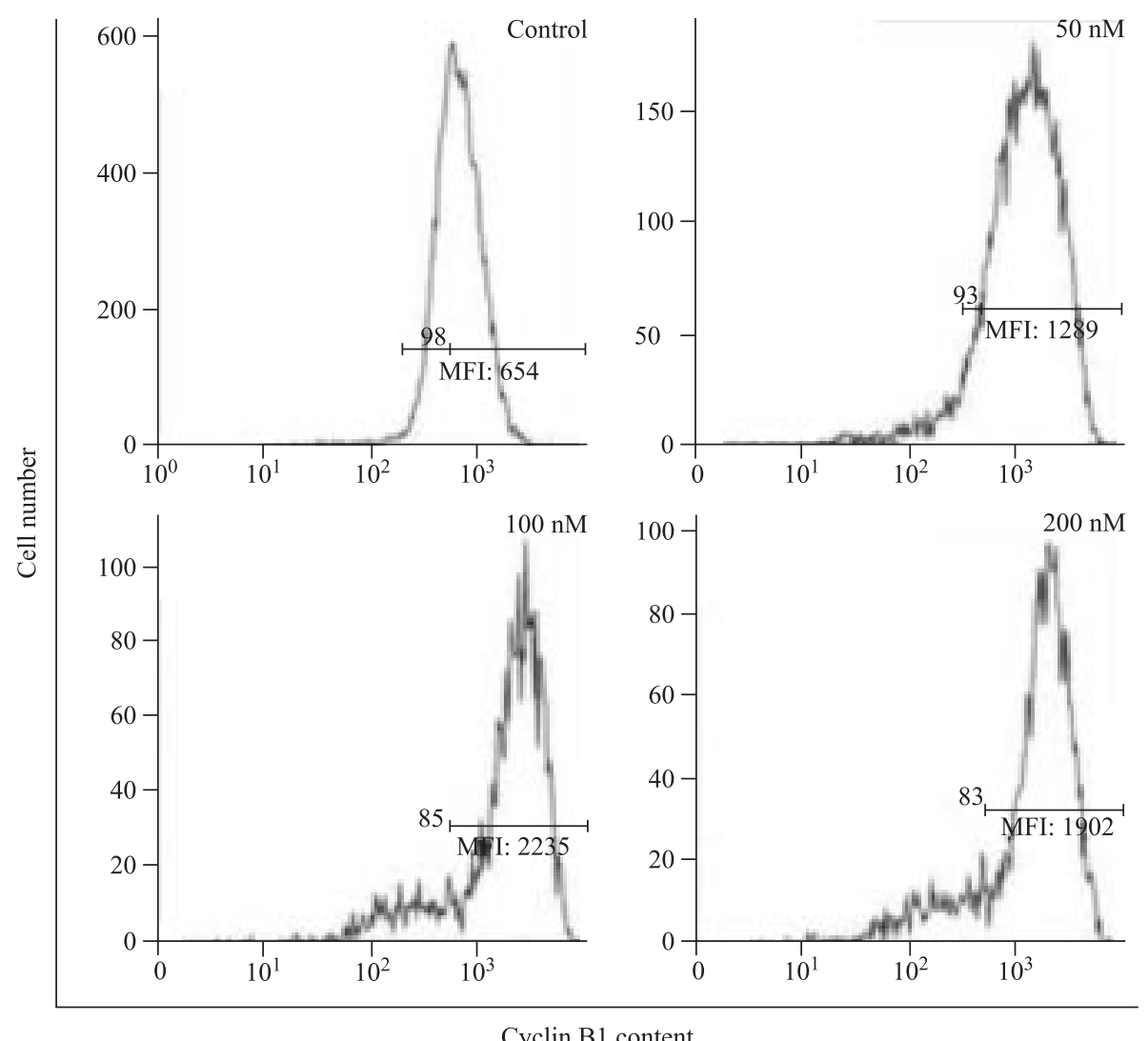

Figure 8. Flow cytometry analysis of cyclin B1 content in A549 treated with doxorubicin. Representative histograms of five independent experiments are shown: upper left — control; upper right $-50 \mathrm{nM}$ of doxorubicin; lower left $-100 \mathrm{nM}$ of doxorubicin; and lower right $-200 \mathrm{nM}$ of doxorubicin. The percentage of cells with cyclin B1 expression is shown on horizontal lengths; MFI - mean fluorescence intensity

\section{Cyclin B1}

Cytometric analysis of cyclin B1 showed that the percentage of cyclin B1-positive cells decreased gradually after treatment with increasing concentrations of doxorubicin, which is consistent with the results of cell viability obtained in our conditions. As regards a level of cyclin B1 in the cyclin-positive cells, we observed an increase in the mean fluorescence intensity of this protein (Figure 8). Additionally, confocal microscopic examination showed cytoplasmic and nuclear localization of cyclin B1. In control cells, cytoplasmic localization was more prominent, whereas in the cells exposed to doxorubicin, cyclin B1 was more abundant in the nucleus area (Figure 9).

\section{Discussion}

The aim of this work was to evaluate the level of mitotic cyclin B1 in the context of senescence and cell death. A previous study was performed in which an unstable senescence-like program was shown to be induced in A549 cells after treatment with similar concentrations of doxorubicin [6].
On the other hand, in the present research, we wanted to verify if a longer recovery time could result in a more stable and effective senescence process. As in the previous studies, we observed an induction of different kinds of death and senescence features in the population of cells exposed to doxorubicin. After the doxorubicin treatment, there was a visible increase in the percentage of cells typical of $\mathrm{G} 2 / \mathrm{M}$ phases of the cell cycle, but also an increased polyploidy, which may be indicative of mitotic catastrophe execution.

At the highest dose of the drug, the number of endocycling cells decreased, which may reflect either a more effective G2/M arrest or an increased percentage of cells dying due to mitotic catastrophe. Previously, an increase in the percentage of cells in the $\mathrm{G} 2 / \mathrm{M}$ phase of the cell cycle, as well as an increase in the number of cells with more G2/M content, was observed (50 and $100 \mathrm{nM}$ of doxorubicin); however, at $200 \mathrm{nM}$ of doxorubicin, there occurred a visible decrease in the number of cells with more G2/M DNA content in comparison with 50 and $100 \mathrm{nM}$ of dox (which was confirmed statistically by Tuckey's multiple comparison test, $\mathrm{p}<0.001$ ). 


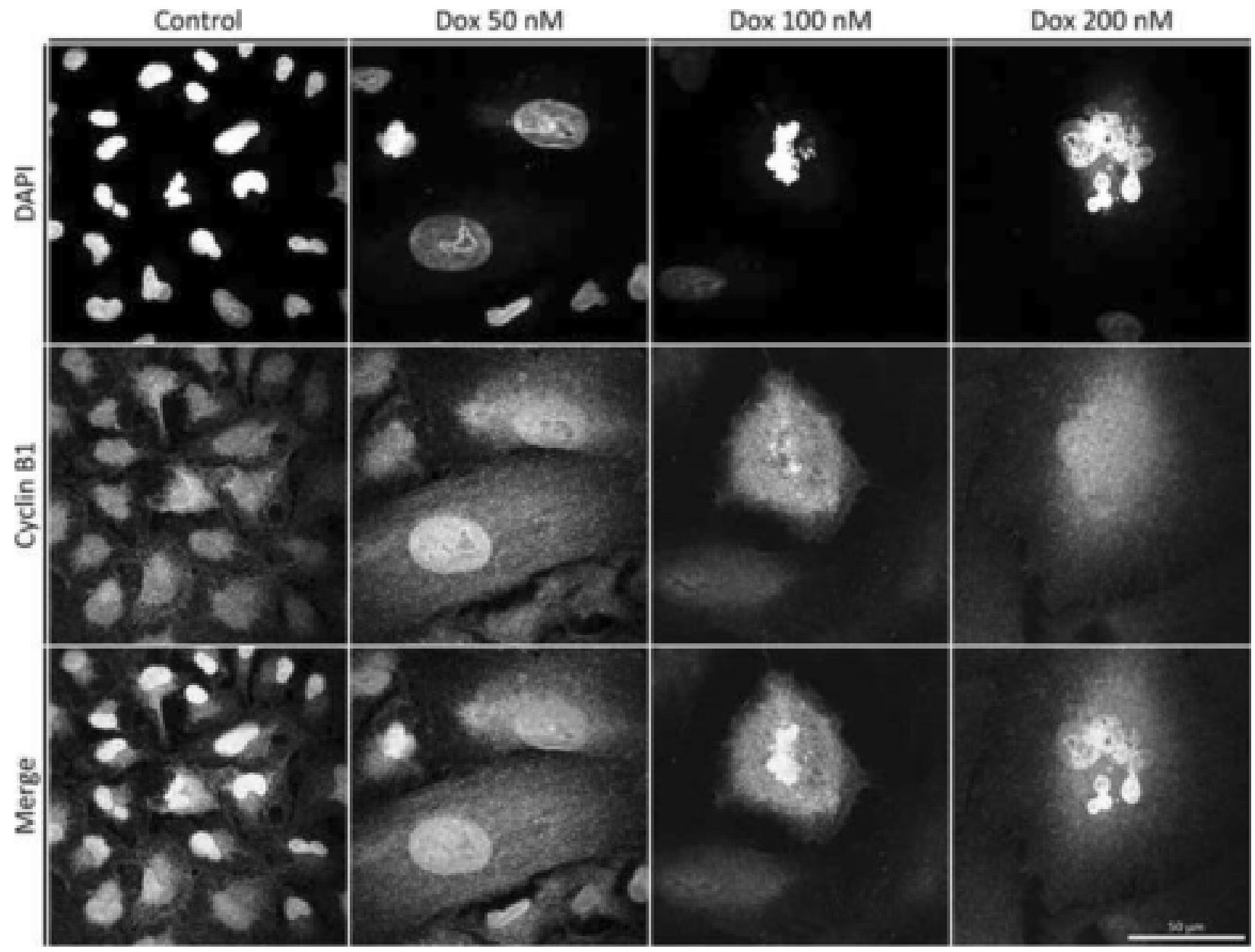

Figure 9. Confocal microscopic examination of cytoplasmic and nuclear localization of cyclin B1 in A549 cells treated with doxorubicin. Cells were treated with 50, 100, and $200 \mathrm{nM}$ doxorubicin and immunolabeled for the presence of cyclin B1. Cell nuclei were labeled with DAPI

Nevertheless, as regards a stable cell cycle arrest, it seems that senescence associated heterochromatin foci, which would be related to a permanent senescence program, did not appear. Instead, at the highest dose of doxorubicin, there was also the highest percentage of TUNEL-positive cells. An increased fraction of TUNEL-positive cells at the lowest drug concentration $(50 \mathrm{nM})$ may reflect the presence of DNA strand breaks that are non-lethal and, as a result, some of these cells may recover. This assumption was further confirmed by the evaluation of viability, which revealed a dose-dependent decrease in living cells fraction. Therefore it seems that the highest percentage of cells undergoing repair was observed at a $50 \mathrm{nM}$ concentration of doxorubicin, whereas the highest concentration resulted in the induction of mitotic catastrophe.

In the present study, we observed an increase in the fluorescence intensity of cyclin B1. Perhaps this was related to a transiently elevated level of this protein as a consequence of mitotic catastrophe execu- tion after a low-dose doxorubicin treatment. It was previously revealed that treatment with different concentrations of doxorubicin may have various effects on cancer Huh-7 cells as regards a cell death mode as well as levels of cyclin A and cyclin B [12]. Moreover, this study showed that the decision concerning cell death modality is strictly correlated with $\mathrm{Cdk} 1$ and Cdk2 activities. These seemed to be crucial for the execution of mitotic catastrophe [12]. It has been suggested that polyploidization associated with the senescent cell morphology appearing after DNA damage may result from degradation of cyclin B1 [13]. Cell cycle arrest may also be accompanied by alterations in cyclin B1 level. For example, a decrease in cyclin B1 levels was observed during hyperoxic stress in A549 cells, which resulted in growth inhibition. This effect was explained by increased proteolysis of cyclin B1 or, alternatively, by the action of inhibitory proteins, like $\mathrm{p} 21$ or $14-3-3 \delta$, which may act directly on the Cdk1-cyclin B1 complex [14]. 
On the other hand, Dan and Yamori [15] suggested that changes in the level of cyclin B1 and G2/M arrest in A549 cells exposed to doxorubicin are regulated in the manner independent of p53, because similar changes were not induced by cisplatin, a well-known p53 activator [15]. In agreement with that, it was shown that an increased level of cyclin B1 may appear in some G2/M-arrested cancer cells with defects in p53 [16]. However, we could not exclude that the increase in the cyclin level observed by us may be strictly correlated with morphological changes appearing in this population of cells after treatment with doxorubicin.

Cyclin B1, apart from other cyclins, is often considered as a potential prognostic marker in different diseases, particularly in cancer [17-21]. For example, it has been shown that cyclin B1 increases during mitotic catastrophe execution induced by different pharmacological or genetic factors. This has been described, for example, in colon cancer cells exposed to doxorubicin [22].

One of the causes of mitotic catastrophe execution may be premature entry into mitosis before the completion of DNA replication, which results in abnormal organization of chromosomes in the presence of the high activity of cyclin B1/Cdk1 complex in the nucleus area [11,23]. There are suggestions that such an aberrant mitosis is mediated by Notch inhibition followed by NF- $\kappa$ B activity, which in turn induces high levels of cyclin B1 [24]. Increased Cdk1/ /cyclin B1 activity seems also to be crucial for efficient cell killing in cases of apoptosis [25, 26]. In accord with that, it has been shown that nuclear accumulation of cyclin B1 is one of the factors involved in triggering this kind of cell death, as it was found mainly in the cytoplasm of the cells that were apoptotic-resistant and accumulated in the nucleus of the apoptotic-prone ones [27]. Moreover, it has been proven that targeting cyclin B1 protein by RNAi or chemical inhibitors leads to decreased number of apoptotic HT29 cells after treatment with camptothecin [23]. On the other hand, reduction of cyclin B1 level may also induce cell cycle arrest and apoptosis in some cancer cells [28].

In our study, we observed that a longer recovery time was related to an increased number of $\mathrm{G} 2 / \mathrm{M}$ arrested cells. However, apart from these cells, the fraction of polyploid cells was still quite high. Only at the highest dose of the drug might a cell cycle arrest be more efficient, as indicated by increased G2/M content in comparison with poliploidy.

It is tempting to hypothesize that this could reflect a more stable senescence program. Concomitantly, at $200 \mathrm{nM}$ doxorubicin, the level of cyclin B1 seemed to decrease compared to $100 \mathrm{nM}$. These changes in cyclin B1 levels may suggest that this protein is differently regulated at mitotic catastrophe to senescence transition.

\section{Acknowledgements}

This work was support by the Nicolaus Copernicus University, Collegium Medicum (grant 17/2009, and grant 586/2010).

\section{References}

1. Pines $\mathrm{J}$, Hunter T. Human cyclin $\mathrm{A}$ is adenovirus E1A associated protein $\mathrm{p} 60$ and behaves differently from cyclin B. Nature. 1990;346:760-763.

2. Innocente SA, Abrahamson JLA, Cogswell JP, Lee JM. p53 regulates a G2 checkpoint through cyclin B1. Proc Natl Acad Sci. 1999;96:2147-2152.

3. Halloway SL, Glotzer M, King RW, Murray AW. Anaphase is initiated by proteolysis rather than by the inactivation of maturation-promoting factor. Cell. 1993;73:1393-1402.

4. Sugrue MM, Shin DY, Lee SW, Aaronson SA. Wild-type p53 triggers a rapid senescence program in human tumor cells lacking functional p53. Proc Natl Acad Sci. 1997; 94: 9648-9653.

5. Blagosklonny MV. Cell senescence and hypermitogenic arrest. EMBO reports. 2003;4: 358-362.

6. Litwiniec A, Grzanka A, Helmin-Basa A, Gackowska L, Grzanka D. Features of senescence and cell death induced by doxorubicin in A549 cells: organization and level of selected cytoskeletal proteins. J Cancer Res Clin Oncol. 2010;136:717-736.

7. Dimri GP, Lee $X$, Basile $G$ et al. A biomarker that identifies senescent human cells in culture and in aging skin in vivo. Proc Natl Acad Sci USA. 1995;92:9363-9367.

8. Zhang R, Chen W, Adamus PD. Molecular dissection of formation of senescence-associated heterochromatin foci. Mol Cell Biol. 2007;27:2343-2358.

9. Schmitt CA. Cellular senescence and cancer treatment. Biochim Biophys Acta. 2007;1775; 5-20.

10. Roninson IB. Tumor cell senescence in cancer treatment. Cancer Research. 2003;63: 2705-2715.

11. Chen B, Cheng M, Hong DJ, Sun FY, Zhu CQ. Okadaic induced cyclin B1 expression and mitotic catastrophe in rat cortex. Neuroscience Letters. 2006;406:178-182.

12. Park SS, Eom YW, Choi KS. Cdc2 and Cdk2 play critical roles in low dose doxorubicin-induced cell death through mitotic catastrophe but not in high dose doxorubicin-induced apoptosis. Bioch Biophys Res Commun. 2005; 334:1014-1021.

13. Kikuchi I, Nakayama Y, Morinaga T, Fukumoto Yamaguchi N. A decrease in cyclin B1 levels leads to polyploidization in DNA damage-induced senescence. Cell Biol Int. 2010;34:645-653.

14. McGrath-Morrow SA, Stahl J. Growth arrest in A549 cells during hyperoxic stress is associated with decreased cyclin B1 and increased p21 Waf1/Cip1/Sdi1 levels. Bioch Biophys Acta. 2001;1538:90-97.

15. Dan S, Yamori T. Repression of cyclin B1 expression after treatment with adriamycin, but not cisplatin in human lung cancer A549 cells. Bioch Biophys Res Commun. 2001;280: 861-867. 
16. Cerquetti L, Bucci B, Marchese R et al. Mitotane increases the radiotherapy inhibitory effect and induces $\mathrm{G}_{2}$-arrest in combined treatment on both H295R and SW13 adrenocortical cell lines. Endocr Relat Cancer. 2008;15:623-634.

17. Aaltomaa S, Lipponen P, Ala-Opas $\mathrm{M}$ et al. Expression of cyclins $A$ and D and p21 (waf 1/cip1) proteins in renal cell cancer and their relation to clinicopathological variables and patient survival. Br J Cancer. 1999;80:2001-2007.

18. Nimeus-Malmström E, Koliadi A, Ahlin C et al. Cyclin B1 is a prognostic proliferation marker with a high reproducibility in a population-based lymph node negative breast cancer cohort. Int J Cancer. 2010;127:961-967.

19. Kosacka M, Korzeniewska A, Jankowska R. Attempt to assess the prognostic significance of the expression of cyclin B1 in patients operated on for stage I-IIIA non small cell lung cancer - preliminary report. Pol Merk Lek. 2010;164; 117-121.

20. Long H, Gao X, Chen X et al. The significance of cyclin B1 expression in colorectal cancers. Chin J Clin Oncol. 2006;5:399-401.

21. Yasuda $\mathrm{M}$, Takesue $\mathrm{F}$, Inutsuka $\mathrm{S}$ et al. Overexpression of cyclin B1 in gastric cancer and its clinicopathological signifi- cance: an immunohistological study. J Cancer Res Clin Oncol. 2002;128:412-416.

22. Chan TA, Hermeking H, Lengauer C, Kinzler KW, Vogelstein B. 14-3-3sigma is required to prevent mitotic catastrophy after DNA damage. Nature. 1999;401:616-620.

23. Borgne A, Versteege I, Mahe M et al. Analysis of cyclin B1 and CDK activity during apoptosis induced by camptothecin treatment. Oncogene. 2006;25:7361-7372.

24. Curry CL, Reed LL, Broude E et al. Notch inhibition in Kaposi's sarcoma tumor cells leads to mitotic catastrophe through nuclear factor-?B signaling. Mol Cancer Ther. 2007;6:1983-1992.

25. Castedo M, Perfettini JL, Roumier T, Kroemer G. Cyclin-dependent kinase-1: linking apoptosis of cell cycle and mitotic catastrophe. Cell Death and Differentiation . 2002;9:1287-1293.

26. Castedo M, Perfettini JL, Roumier T et al. Cell death by mitotic catastrophe: a molecular definition. Oncogene. 2004;23:2825-2837.

27. Porter LA. Nuclear localization of cyclin B1 regulates DNA damage-induced apoptosis. Blood. 2003;101:1928-1933.

28. Yuan J, Yan R, Krämer A et al. Cyclin B1 depletion inhibits proliferation and induces apoptosis in human tumor cells. Oncogene. 2004;23:5843-5852. 